\title{
Bone mineral density in adolescent female athletes: relationship to exercise type and muscle strength
}

\author{
CRAIG S. DUNCAN, CAMERON J. R. BLIMKIE, CHRISTOPHER T. COWELL, STEPHEN T. BURKE, \\ JULIE N. BRIODY, and ROBERT HOWMAN-GILES
}

University of Sydney, Sydney, AUSTRALIA; Australian Catholic University, Sydney, AUSTRALIA; New Children's Hospital, Sydney, AUSTRALIA; and McMaster University, Hamilton, Ontario, CANADA

\begin{abstract}
DUNCAN, C. S., C. J. R. BLIMKIE, C. T. COWELL, S. T. BURKE, J. N. BRIODY, and R. HOWMAN-GILES. Bone mineral density (BMD) in adolescent female athletes: relationship to exercise type and muscle strength. Med. Sci. Sports Exerc., Vol. 34, No. 2, pp. 286-294, 2002. Purpose: This study investigated the influence of different exercise types and differences in anatomical distribution of mechanical loading patterns on bone mineral density (BMD) in elite female cyclists, runners, swimmers, triathletes, and controls $(N$ $=15$ per group). Associations between leg strength and BMD were also examined. Methods: Areal BMD $\left(\mathrm{g} \cdot \mathrm{cm}^{-2}\right)$ was assessed by duel-energy x-ray absorptiometry (DXA) (total body (TB), lumbar spine (LS), femoral neck (FN), legs, and arms). Right knee flexion and extension strength was measured using a Cybex Norm isokinetic dynamometer at $60^{\circ} \cdot \mathrm{s}^{-1}$. Results: Runners had significantly higher unadjusted TB, LS, FN, and leg BMD than controls $(P<0.05)$; higher TB, FN, and leg BMD than swimmers $(P<0.05)$; and greater leg BMD than cyclists $(P<0.05)$. Absolute knee extension strength was significantly $(P<0.01)$ correlated $(0.33 \leq \mathrm{r} \leq 0.44)$ with TB, FN, LS, and leg BMD for all groups combined. Weaker but still significant correlations $(0.28 \leq \mathrm{r} \leq 0.33)$ existed for normalized (per leg lean tissue mass) knee extension strength and all BMD sites, except FN BMD. There were no significant correlations between absolute or normalized knee flexion strength and any of the BMD variables. Absolute knee extension strength was entered as the second independent predictor for LS and leg BMD in stepwise multiple linear regression analysis (MLRA), accounting for increments of $4 \%$ and $12 \%$, respectively, in total explained variation. Conclusion: We conclude that running, a weight bearing exercise, is associated with larger site-specific BMD than swimming or cycling, that the generalized anatomical distribution of loads in triathlon appears not to significantly enhance total body BMD status, and that knee extension strength is only a weak correlate and independent predictor of BMD in adolescent females. Key Words: MECHANICAL LOADING, ELITE SPORT, WEIGHT BEARING, NONWEIGHT BEARING
\end{abstract}

$\mathrm{T}$ The influences of exercise and mechanical loading on the skeleton have been extensively researched in both animals and humans (e.g., Bourrin et al. (4) and Heinonen et al. (14)). Exercise type appears to be an important variable influencing the osteogenic adaptive response in animals. Weight bearing loads that are characterized by high axial compressive forces have been identified as having the most positive influence on bone mineral density (BMD) in rodents (4). In humans, the effect of exercise type on skeletal adaptations has been inferred mostly from cross-sectional studies of athletes from different sports, compared with nonathlete controls. These results suggest that weight bearing sports (10) are generally more osteogenic than nonweight bearing (14) activities. Skeletal adaptations to loading also appear to be site-specific $(4,17)$, suggesting that the anatomical distribution of loads may be another important factor influencing both regional and total peak bone mass.

0195-9131/02/3402-0286/ $\$ 3.00 / 0$

MEDICINE \& SCIENCE IN SPORTS \& EXERCISE ${ }_{\circledast}$

Copyright (C) 2002 by the American College of Sports Medicine

Submitted for publication January 2001

Accepted for publication May 2001.
Several studies, differing in subject characteristics and sport specialization (e.g., Courteix et al. (7) and Nordstrom et al. (23)) have compared BMD in children and adolescents involved in either weight bearing or nonweight bearing exercise with nonexercising controls. To our knowledge, there are no comparative studies of BMD among sports that are predominantly weight bearing or nonweight bearing, or which include combinations of both types of loading characteristics while also controlling for other putative osteogenic influences, such as years of prior training and maturity status, in children or adolescent females. Consequently, there is little valid information about the relative osteogenic effects of either the different types of loading conditions (weight bearing vs nonweight bearing vs their combined influence) or the importance of the anatomical distribution (regional vs general) of the loading patterns inherent in various sports, for optimization of skeletal development in these populations.

Muscle contraction force acting directly or indirectly on bone has also been implicated as an important determinant of skeletal adaptation to exercise (5). Some (29) but not all studies $(25,28)$ have reported positive associations between muscle strength and BMD in children and adults. Highintensity strength training programs involving adolescent 
(2) and young adult females (30) have reported either no or very small significant increases in BMD, suggesting either a nonexistent, weak, or temporally dissociated relationship between strength and BMD in these populations. Recent studies have also suggested stronger bone mass-muscle strength relationships in untrained compared with trained adolescent males (24), with a reversed association in adolescent females (1). Additionally, a recent study (28) has suggested that the lean body mass and, by proxy, the muscle strength-BMD relationship may differ between males and females during the peripubertal and adolescent years. The muscle strength-BMD relationship remains relatively underinvestigated in children in general, and adolescent females in particular, and the mechanisms underlying this putative relationship are generally poorly understood and remain to be elucidated.

The paucity of research in these areas for adolescent females is surprising for several reasons. First, increased exercise during adolescence may represent the final opportunity before skeletal consolidation for substantially augmenting peak bone mass, an identified risk factor for osteoporosis in females (20). Second, activity participation rates and sport preferences change dramatically in females during the transition from prepuberty to adolescence (26), and information regarding the potential osteogenic benefits of different types of exercise might be useful in promoting increased exercise and guiding personal activity choices during this transition period. Finally, since most females attain their peak muscle strength during adolescence (3), a clearer understanding of the osteogenic importance of muscle strength in this population is clearly required.

The primary purposes of this study were to 1) compare BMD among athletes who specialize in predominantly weight bearing, nonweight bearing, or combinations of both types of sports; and 2) to investigate the relationship between anatomical distribution of sport specific mechanical loading patterns and BMD in adolescent female athletes. A secondary purpose was to describe the relationship between regional BMD and muscle strength of the legs. We hypothesized that lower extremity regional BMD would be higher in athletes whose sports included predominantly weight bearing (runners and triathletes) compared with nonweight bearing (swimmers and cyclists) loading, and that total body BMD would be higher in triathletes exposed to more generalized anatomical loading conditions with varied strain characteristics, compared with athletes from sports with a more regional and less varied strain distribution pattern. We also hypothesized positive associations between absolute leg muscle strength and BMD for all groups combined, with weaker associations for normalized strength (per leg lean tissue mass (LTM)).

A cross-sectional study design incorporating an elite athlete model was used, which compared BMD in triathletes with athletes who specialized in swimming, cycling, or running. Triathlon, a new Olympic sport consisting of swimming, cycling, and running events, involves both weight bearing and nonweight bearing exercise and a diverse anatomical load distribution pattern. With this model, we are able to compare differences in BMD between weight bearing (running) and nonweight bearing (swimming and cycling) sports, with a sport that incorporates both types of loading conditions (triathlon). Additionally, this model allows for comparisons of associations between region-specific loading patterns and BMD in the single-discipline sports (swimming, cycling, and running), with the more generalized loading patterns in the multidiscipline sport of triathlon.

\section{METHODS}

\section{Study Participants}

Seventy-five adolescent female volunteers (15-18 yr of age) were included in this study. Groups consisted of swimmers, cyclists, runners, triathletes, and nonathlete controls ( $N=15$ per group). All athletes specialized in endurance events and were either state or national level representatives. Athletes participated in at least $8 \mathrm{~h} \cdot \mathrm{wk}^{-1}$ of training and competition during the previous year. Controls currently were involved in less than $2 \mathrm{~h} \cdot \mathrm{wk}^{-1}$ of physical activity and previously had not been involved in elite competitive sport. All subjects were Caucasian, and girls were postmenarcheal by at least $1 \mathrm{yr}$, having had 11 or more menstrual cycles during the previous 12 months. None of the subjects had previously or were currently taking oral contraceptive medication.

This study was approved jointly by the Human Ethics Committee of the University of Sydney and the New Children's Hospital, Sydney. An investigator explained the procedures and requirements, and then each participant gave written informed consent.

\section{Procedures}

Participants were required to make one visit to our laboratory for a period of $3 \mathrm{~h}$. During this time, BMD, anthropometry, strength, general health, and physical activity were assessed by a single investigator. All procedures were conducted at the New Children's Hospital, Sydney, and the Children's Hospital Institute of Sports Medicine, which adjoins the hospital. Measurements were completed within 1 month of the respective sports' national championships, to coincide with peak training loads.

BMD. Areal BMD $\left(\mathrm{g} \cdot \mathrm{cm}^{-2}\right)$ was measured for the total body (TB), lumbar spine (LS), and right femoral neck (FN) by dual-energy x-ray absorptiometry (DXA), using a Lunar scanner (Model DPX, pencil beam; Lunar Radiation Corp., Madison, WI) and standard operating procedures (19). The TB scan was performed with a fast scan speed, whereas the medium speed option was used for the LS and FN. The same investigator completed and analyzed all scans using standard analysis protocols. Regional BMD analyses of the legs, arms, and skull were obtained from the TB scan. Participants wore light clothing without zippers or buttons and removed all jewellery for the scanning procedures. A spine phantom provided by Lunar Radiation Corp. was used daily throughout the study period for quality control assessment. 
The coefficient of variation $(\mathrm{CV})$ in our laboratory was $0.67 \%$ for the phantom spine BMD. A TB phantom (constructed of aluminum strips and rice bags mounted together, as recommended by Lunar Radiation Corp.) was also used for quality control during the study period. The CV for this total body phantom BMD was $1.1 \%$. In vivo $\mathrm{CV}$ was also calculated by performing TB scans and regional analysis on an adult female for 6 consecutive days. The CVs were $1.20 \%, 1.06 \%, 1.50 \%, 2.2 \%$, and $2.4 \%$ for TB, LS, FN, leg, and arm areal BMD, respectively (20).

Anthropometry. Body weight was measured on an electronic scale accurate to $500 \mathrm{~g}$ (Wedderburn, Sydney, Australia) with subjects dressed in light clothing and without shoes. Standing height was measured with a standard stadiometer (Wedderburn). Percent body fat and lean tissue mass (LTM) were derived from the total body DXA scan. The CV for LTM is $1.1 \%$ in our center (20). LTM of the legs was derived from regional analysis of the total body DXA scans.

Strength. Strength was measured with a Cybex Norm isokinetic dynamometer (Lumex, Inc., Ronkonkoma, NY). Isokinetic knee extension and flexion torque of the right leg was measured at $60^{\circ} \cdot \mathrm{s}^{-1}$ with standard positioning and stabilizing procedures for the legs and torso. The protocol involved six warm-up repetitions followed by a 1-min recovery and three maximal continuous flexion and extension repetitions. The average torque of these maximal repetitions was used as the criterion strength measure. The short-term reliability of isokinetic strength testing is good, with CVs between $5 \%$ and $11 \%$ when measurements are made by the same tester (25). Strength measures are presented and analyzed both in absolute $(\mathrm{Nm})$ and relative terms $\left(\mathrm{Nm} \cdot \mathrm{g}^{-1}\right)$, normalized for leg LTM.

Physical activity, training, and medical histories. A physical activity questionnaire (NSW Schools Physical Activity Questionnaire, 1998) was completed on the day of testing to determine the activity levels of controls. This questionnaire solicited information on the frequency, type, and duration of activities over the previous year. Athletes were required to record their training history for a period of 2 wk after testing. Sport specific (e.g., swimming, running, cycling) and total training hours, including supplemental forms of training, were recorded. Athletes were given verbal and written instructions on how to complete this diary. A medical history questionnaire was used to identify menstrual status, cigarette consumption, and medication usage. The questionnaire was completed at the time of testing with the aid of an investigator.

\section{Statistical Analysis}

A one-way ANOVA was used to determine if significant $(P<0.05)$ differences existed among groups for descriptive, BMD, and strength variables. If the $F$-ratio was significant, differences among groups were subsequently identified using a Tukey post hoc analysis. BMD data were also analyzed using ANCOVA, with adjustments made for the combined influences of LTM, years since menarche (YSM), and years of prior sport specific training (YPT), as these variables differed significantly among groups. Bivariate relationships between $\mathrm{BMD}$, selected descriptive characteristics, and knee extension and flexion strength were determined by correlation analysis (Pearson correlation coefficient) for all groups combined. No attempt was made to compare these relationships between control $(N=15)$ and athlete groups combined $(N=60)$ because of the large discrepancy in samples sizes. An additional comparison of leg BMD was made using ANOVA among the upper, combined middle, and lower quartile groups for normalized (for leg LTM) knee flexion and extension strength for all groups combined. Lastly, a series of stepwise multiple linear regression analyses (MLRA) incorporating descriptive and strength variables that were significantly correlated with BMD in the bivariate analyses were performed to determine significant predictors of BMD at all sites for all groups combined. Correlations among variables and differences between groups were considered significant at $P<0.05$.

Group sample size was determined on the basis of an estimated conservative effect size (parameter difference) of 0.5 , calculated as the SD of possible group means divided by an estimate of the SD of the BMD measurement, a power of 0.80 , and an alpha of 0.05 (9). On the basis of these criteria, samples of 15 subjects per group were required. Observed power values were greater than 0.80 for all but arm BMD, with a power of 0.40 . The statistical software program SPSS version 8.0 (SPSS, Inc., Chicago, IL) was used for all data analysis.

\section{RESULTS}

Descriptive characteristics. Descriptive characteristics of subjects are summarized in Table 1. There were no significant differences among groups for any of the physical variables except for lean tissue mass. Cyclists had significantly greater lean tissue mass than controls. There were no differences in age at menarche, but runners had a greater number of years postmenarche (YSM) than cyclists. The training history characteristics are also presented in Table 1. Runners trained significantly fewer hours per week than the other sport groups, and swimmers and runners had been involved in specialized training (YPT) significantly longer than cyclists and triathletes.

BMD. Unadjusted areal BMD results are summarized in Figure 1 as box-and-whiskers plots. Runners had significantly higher $(P<0.05)$ BMD than controls for TB $(8.6 \%)$, LS (12.2\%), FN (9.7\%), and leg (13.2\%); and higher TB $(9.2 \%), \mathrm{FN}(10 \%)$, and leg (13.2\%) BMD than swimmers. Runners also had significantly higher $(P<0.05)$ BMD for the legs $(11 \%)$ compared with the cyclists. There were no significant differences between runners and triathletes. After adjusting for the combined influence of LTM, YSM, and YPT, runners had significantly higher TB BMD than swimmers and cyclists, higher LS BMD than triathletes, and higher FN and leg BMD than all other groups (Table 2). Triathletes also had significantly higher adjusted leg BMD than swimmers, but there were no other significant differ- 
TABLE 1. Descriptive characteristics of subjects ( $N=15$ per group).

\begin{tabular}{|c|c|c|c|c|c|}
\hline & Controls & Swimmers & Cyclists & Runners & Triathletes \\
\hline Age & $16.9(0.9)$ & $16.7(1.3)$ & $16.5(1.4)$ & $17.6(1.4)$ & $17.7(1.1)$ \\
\hline Height (m) & $1.66(0.07)$ & $1.67(.06)$ & $1.66(.04)$ & $1.68(.04)$ & $1.67(.04)$ \\
\hline Weight (kg) & $57.8(10.5)$ & $58.6(7.7)$ & $60.3(7.6)$ & $60.7(6.4)$ & $59.4(5.9)$ \\
\hline Body fat $(\%)$ & $31.3(10.2)$ & $27.1(6.0)$ & $23.6(8.6)$ & $25.3(5.3)$ & $26.3(6.2)$ \\
\hline Lean tissue $(\mathrm{kg})$ & $37.4(5.1)$ & $41.3(5.3)$ & $43.8(5.2)^{b}$ & $42.6(6.0)$ & $42.4(3.6)$ \\
\hline Age at menarche & $13.5(1.3)$ & $13.7(1.4)$ & $13.6(0.9)$ & $13.4(0.9)$ & $13.5(0.9)$ \\
\hline Menstrual cycles per yr & $12.4(1.6)$ & $12(0.1)$ & $12.2(0.2)$ & $12.3(1.8)$ & $11.9(0.2)$ \\
\hline Yr of specialized training & - & $6.1(2.7)^{c, d}$ & $3.1(1.8)$ & $5.0(1.6)^{c, e}$ & $2.5(1.2)$ \\
\hline${ }^{2} \mathrm{Yr}$ of total training & - & $6.9(2.3)$ & $6.1(2.0)$ & $6.2(1.7)$ & $7.4(2.2)$ \\
\hline
\end{tabular}

Values are mean \pm SD.

${ }^{a}$ Years of total training includes years of specialized training in the various sports by which subjects were categorized for the purpose of this study, plus additional years of participation in other sports prior to becoming sport specialists.

${ }^{b}$ Significantly greater than controls, $P<0.05$.

${ }^{c}$ Significantly greater than cyclists, $P<0.05$.

${ }^{d}$ Significantly greater than runners, $P<0.01$.

${ }^{e}$ Significantly greater than triathletes, $P<0.01$.

ences for unadjusted or adjusted BMD at any measurement site among swimmers, cyclists, or triathletes. There were no significant differences for either unadjusted or adjusted arm $\mathrm{BMD}$, or for skull BMD (range, $2.03 \pm 0.17$ to $2.15 \pm 0.24$ $\mathrm{g} \cdot \mathrm{cm}^{-2}$ ) among groups.

Bivariate correlations between measures of BMD and selected descriptive variables are presented in Table 3. Body weight and lean tissue mass were significantly correlated with all BMD measures. Age and BMI were significantly
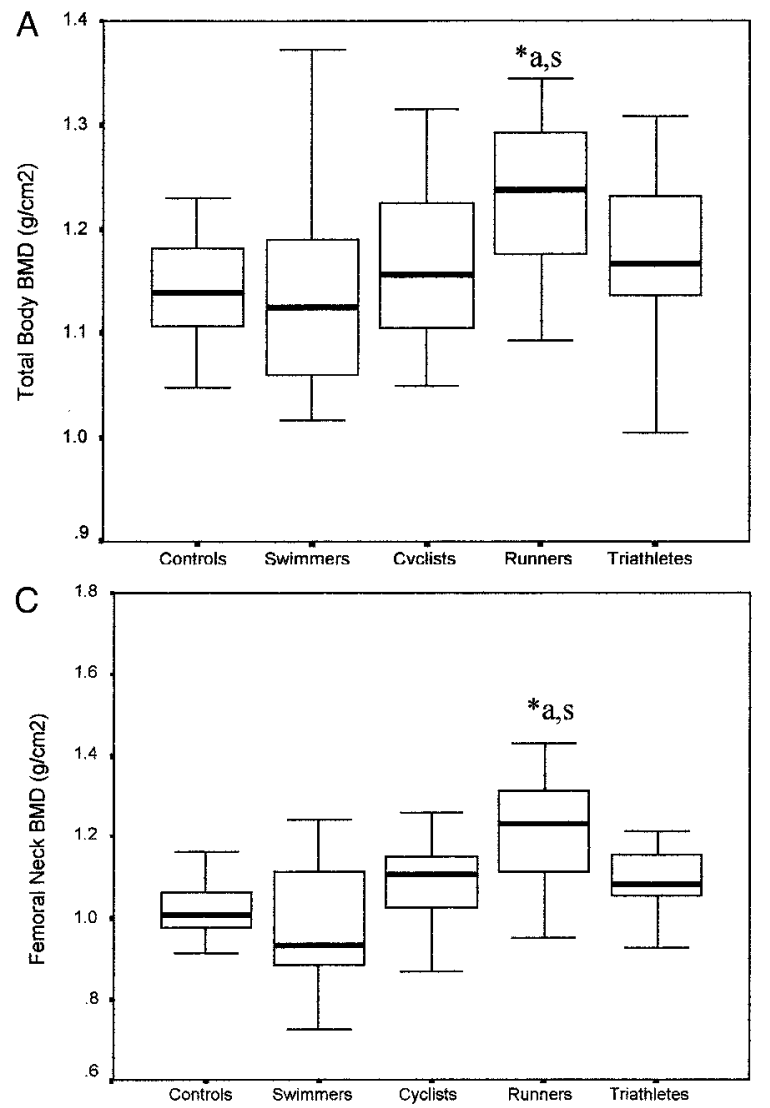

correlated with all BMD measures except FN BMD, and height with all but leg and arm BMD. There were no significant relationships between BMD variables and either percent body fat or menstrual status. Hours of training per week and years of sport specific training were positively correlated with arm BMD only.

Strength. Strength results are summarized in Table 4. Absolute knee extension and flexion strength were significantly higher in cyclists and runners, compared with controls.
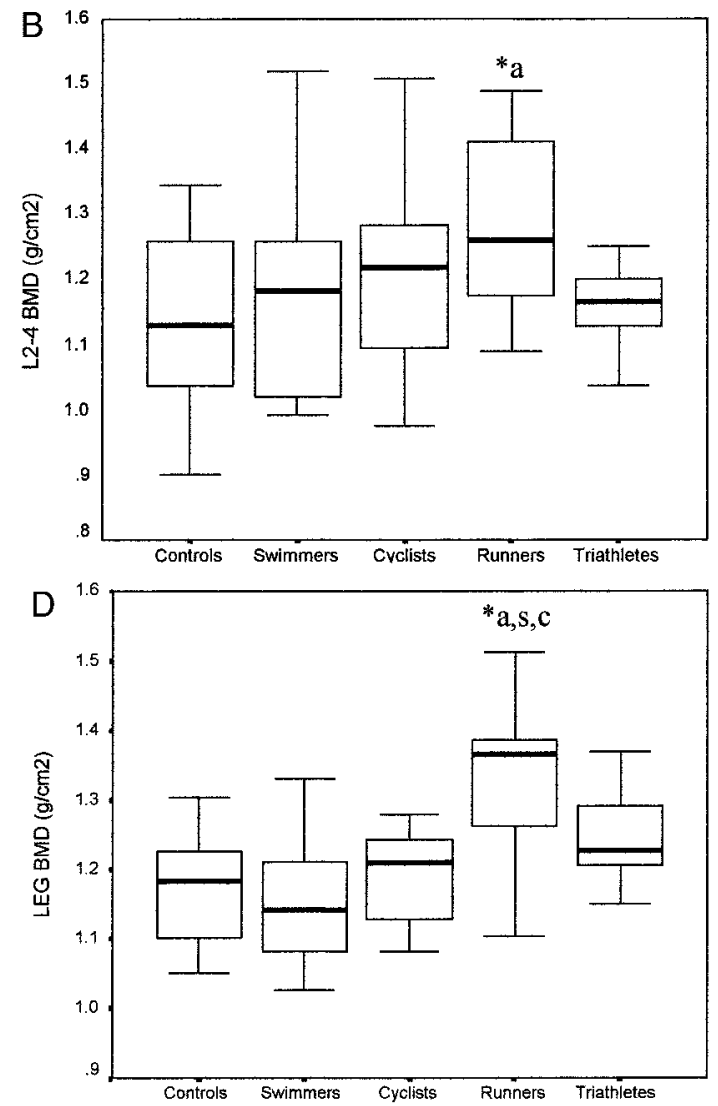

FIGURE 1-A comparison of (A) total body, (B) lumbar spine, (C) femoral neck, and (D) leg BMD among sport groups. Data are presented as box-and-whiskers plots. The horizontal line in the box represents the group median; the upper and lower borders of the box represent the 75th and 25th percentiles, respectively; and the vertical lines represent the largest and smallest observed values that are not outliers. ${ }^{* a}$ Runners significantly greater than controls $(P<\mathbf{0 . 0 5}) .{ }^{*}$ Runners significantly greater than swimmers $\left(P<0.05\right.$. ${ }^{*}$ Runners significantly greater than cyclists $(P<0.05)$. 
TABLE 2. Mean BMD $(95 \% \mathrm{CI})$ adjusted for lean tissue mass, years since menarche, and years of specialized training (ANCOVA).

\begin{tabular}{|c|c|c|c|c|c|}
\hline & Controls & Swimmers & Cyclists & Runners & Triathletes \\
\hline Total body $\left(\mathrm{g} \cdot \mathrm{cm}^{-2}\right)$ & $\begin{array}{c}1.19 \\
(1.1-1.2)\end{array}$ & $\begin{array}{c}1.13 \\
(1.1-1.2)\end{array}$ & $\begin{array}{c}1.16 \\
(1.1-1.2)\end{array}$ & $\begin{array}{l}1.23^{b, c} \\
(1.2-1.3)\end{array}$ & $\begin{array}{c}1.18 \\
(1.1-1.2)\end{array}$ \\
\hline Lumbar spine $\left(\mathrm{g} \cdot \mathrm{cm}^{-2}\right)$ & $\begin{array}{c}1.21 \\
(1.1-1.3)\end{array}$ & $\begin{array}{c}1.18 \\
(1.1-1.3)\end{array}$ & $\begin{array}{c}1.20 \\
(1.1-1.3)\end{array}$ & $\begin{array}{l}1.27^{d} \\
(1.2-1.3)\end{array}$ & $\begin{array}{c}1.15 \\
(1.1-1.2)\end{array}$ \\
\hline Femoral neck $\left(\mathrm{g} \cdot \mathrm{cm}^{-2}\right)$ & $\begin{array}{c}1.05 \\
(0.9-1.1)\end{array}$ & $\begin{array}{c}0.99 \\
(0.9-1.1)\end{array}$ & $\begin{array}{c}1.07 \\
(1.0-1.1)\end{array}$ & $\begin{array}{l}1.20^{a, b, c, d} \\
(1.1-1.3)\end{array}$ & $\begin{array}{c}1.11 \\
(1.0-1.1)\end{array}$ \\
\hline $\operatorname{Leg}\left(\mathrm{g} \cdot \mathrm{cm}^{-2}\right)$ & $\begin{array}{c}1.22 \\
(1.2-1.3)\end{array}$ & $\begin{array}{c}1.15 \\
(1.1-1.2)\end{array}$ & $\begin{array}{c}1.18 \\
(1.1-1.2)\end{array}$ & $\begin{array}{l}1.31^{a, b, c, d} \\
(1.3-1.4)\end{array}$ & $\begin{array}{c}1.24^{b} \\
(1.2-1.3)\end{array}$ \\
\hline $\operatorname{Arm}\left(\mathrm{g} \cdot \mathrm{cm}^{-2}\right)$ & $\begin{array}{c}0.92 \\
(0.9-0.97)\end{array}$ & $\begin{array}{c}0.94 \\
(0.9-1.0)\end{array}$ & $\begin{array}{c}0.93 \\
(0.9-1.0)\end{array}$ & $\begin{array}{l}0.98 \\
(0.9-1.0)\end{array}$ & $\begin{array}{c}0.94 \\
(0.9-1.0)\end{array}$ \\
\hline
\end{tabular}

Values are mean and 95\% confidence intervals.

${ }^{a}$ Significantly greater than controls, $P<0.05$.

${ }^{b}$ Significantly greater than swimmers, $P<0.05$.

${ }^{c}$ Significantly greater than cyclists, $P<0.05$.

${ }^{d}$ Significantly greater than triathletes, $P<0.05$.

There were no other significant differences in absolute strength among groups. Runners also had significantly higher normalized knee extension strength than controls, but there were no other significant differences in either normalized knee extension or flexion strength among groups. For the bivariate correlations, absolute knee extension strength was significantly correlated (r) with TB (0.40), LS (0.44), FN (0.33), and leg (0.39) BMD. Normalized knee extension strength was significantly correlated with all BMD measures except FN BMD, but correlations were consistently weaker than for absolute strength $(0.28 \leq \mathrm{r} \leq 0$. 33). There were no significant correlations for either absolute or normalized knee flexion strength and any of the BMD measures. There were no interquartile group differences in leg BMD for either normalized knee flexion or extension strength (Fig. 2).

Results for the MLRA are summarized in Table 5. Body weight entered as the first significant predictor of TB and LS BMD, whereas lean body mass (LBM) and age entered as the first predictors for FN and leg BMD, respectively. Age entered as an additional independent predictor for TB BMD, whereas absolute knee extension strength entered as the second independent predictor for LS and leg BMD. LBM also entered as a significant predictor of arm BMD. Cumulatively, these variables accounted for $14 \%$ to $33 \%$ of the total explained variance, with knee extension strength contributing increments of only $4 \%$ and $12 \%$ for LS and leg $\mathrm{BMD}$, respectively.

\section{DISCUSSION}

The results from this study in part confirmed our first hypothesis by demonstrating that eumenorrheic elite adolescent female runners who perform predominantly weight bearing activity have superior BMD compared with athletes from predominantly nonweight bearing sports. This study demonstrates the potential positive influence of increased weight bearing exercise on BMD in normally menstruating elite adolescent female runners, compared with athletes from other sports with lesser degrees of weight bearing.

Our findings of higher areal BMD across all measurement sites in runners, compared with all other groups, support previous research on young animals (16) and children, where it has been shown that weight bearing activities were associated with larger BMD or elicited significant positive bone mass adaptations (10). In contrast to these findings, however, a number of studies $(19,22)$ have reported no difference or lower BMD in female adolescent or young adult endurance runners compared with sedentary controls. Results from these latter studies, however, may be biased in that they failed to exclude participants with menstrual irregularities. A recent study (22) that compared runners with variable menstrual status concluded that menstrual dysfunction was strongly associated with decreased BMD. Whether endurance running increases the risk of menstrual dysfunction is beyond the scope of the present investigation; however, only two runners were excluded because of abnormal

TABLE 3. Bivariate relationships between areal BMD and selected descriptive variables for all subjects combined.

\begin{tabular}{|c|c|c|c|c|c|}
\hline & $\begin{array}{c}\text { Total Body } \\
\text { BMD }\end{array}$ & $\begin{array}{c}\text { Lumbar } \\
\text { Spine BMD }\end{array}$ & $\begin{array}{c}\text { Femoral } \\
\text { Neck BMD }\end{array}$ & $\begin{array}{l}\text { Leg } \\
\text { BMD }\end{array}$ & $\begin{array}{l}\text { Arm } \\
\text { BMD }\end{array}$ \\
\hline Weight & $0.49^{a}$ & $0.53^{a}$ & $0.31^{a}$ & $0.37^{a}$ & $0.40^{a}$ \\
\hline Lean tissue mass & $0.43^{a}$ & $0.47^{a}$ & $0.36^{a}$ & $0.37^{a}$ & $0.49^{a}$ \\
\hline Age at menarche & 0.01 & -0.05 & -0.05 & 0.01 & 0.04 \\
\hline Yr since menarche & 0.23 & 0.22 & 0.11 & 0.23 & 0.17 \\
\hline
\end{tabular}

\footnotetext{
${ }^{a}$ Indicates $P<0.01$.
}

${ }^{b}$ Indicates $P<0.05$.

${ }^{c}$ Correlation included sport groups only. 
TABLE 4. Summary of isokinetic absolute $(\mathrm{Nm})$ and normalized $\left(\mathrm{Nm} \cdot \mathrm{g}^{-1}\right.$ leg LTM) knee extension and flexion strength measured at $60^{\circ} \cdot \mathrm{s}^{-1}$.

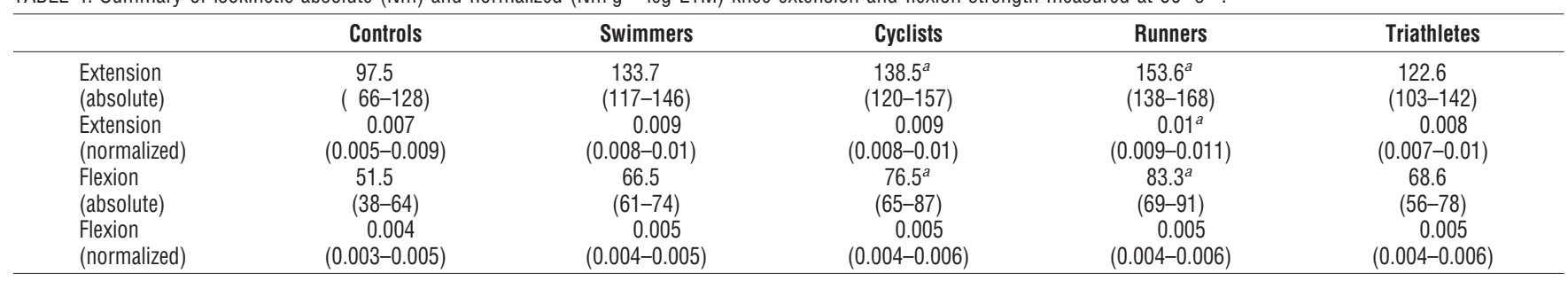

Values are mean and $95 \%$ confidence intervals.

a Significantly greater than controls, $P<0.01$.

menstrual status in our study. Differences in menstrual status, distance specialization, running training volume, and training history may partially explain the discrepancies between our BMD findings and those of prior exercise studies involving female runners.

Our results also suggest that the larger BMD in runners may be achieved with only a modest training volume, since the runners in this study trained only half the hours per week of the other sport groups. Since running is the only activity of the single discipline sports that elicits substantial ground reaction impact forces during weight bearing, it appears that the difference in BMD among runners, swimmers, and cyclists may be explained in large part by this unique loading aspect of this sport. The importance of the weight bearing nature of loading in running is even more evident when BMD results are compared with swimming, a nonweight bearing sport. Despite having begun training several years before puberty, and having the longest sport specific training history of all athlete groups, swimmers had the lowest BMD of all groups, and had values not substantially different from controls. Likewise, the persistence of the trend toward higher BMD at all sites for the runners, after adjustment for YPT, YSM, and LTM, suggests that the nature of the sport specific loading patterns, rather than variability in prior training, menstrual history, or muscle mass, is the most important factor influencing differences in BMD in this investigation.

Triathletes were the only other athlete group whose unadjusted TB BMD values were not significantly inferior to runners and who demonstrated statistically similar TB BMD to runners even after adjustment for YPY, YSM, and LTM. There is little information on bone density in triathletes (26), and to our knowledge this is the first study to report BMD in adolescent female triathletes. Our results are similar to those of Rutherford (26), who reported no difference in TB, LS, or leg BMD between young female adult runners and triathletes. Triathlon is a multidiscipline sport with unique mechanical loading patterns: middle-distance running elicits ground reaction forces and lower body longitudinal compression, bending, and torsional strains on the long bones of the leg, whereas swimming and cycling would elicit predominantly bending and torsional strains on long bones, with no ground reaction forces. The combination of three sport disciplines, with the inclusion of an element of weight bearing, would provide both a broad anatomical distribution and an unusual pattern of strains in triathletes, the latter having been identified as an important determinant of the skeletal adaptive response to mechanical loading in animals (18). With these loading characteristics, we anticipated that triathletes might have had the largest TB BMD and intermediate regional BMD compared with the other athlete groups. The results in the present study do not support our second hypothesis, and suggest that either the strain distribution pattern was not sufficiently novel, or that the strains appear to be of insufficient magnitude to elicit the largest anticipated TB BMD in triathletes.

However, adjusted leg BMD was significantly higher in triathletes compared with swimmers, and with the exception of adjusted LS BMD, there was also a nonsignificant trend $(P=0.08)$ of higher regional unadjusted and adjusted $\mathrm{FN}$
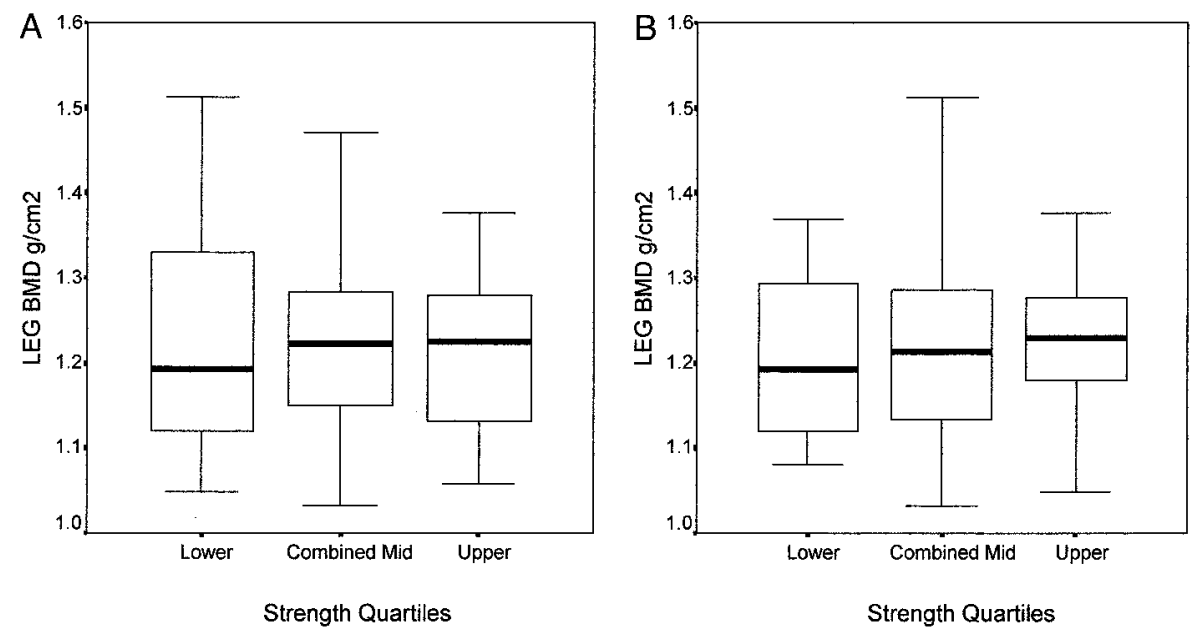

FIGURE 2-A comparison of leg BMD among groups categorized by quartiles for normalized leg (A) flexion and (B) extension strength. Comparisons are made between the upper, combined middle, and lower strength quartiles. Data are presented as box-and-whiskers plots. The horizontal line in the box represents the group median; the upper and lower borders of the box represent the 75th and 25th percentiles, respectively; and the vertical lines represent the largest and smallest observed values that are not outliers. 
TABLE 5. Summary of stepwise multiple linear regression analysis for all groups combined. ${ }^{a}$

\begin{tabular}{|c|c|c|c|c|c|c|c|c|c|c|}
\hline $\begin{array}{l}\text { Combined groups } \\
\text { Included predictors }\end{array}$ & \multicolumn{2}{|l|}{ TB BMD } & \multicolumn{2}{|l|}{ LS BMD } & \multicolumn{2}{|l|}{ FN BMD } & \multicolumn{2}{|l|}{ Leg BMD } & \multicolumn{2}{|l|}{ Arm BMD } \\
\hline & First Weight & 0.47 & First Weight & 0.53 & \multirow{2}{*}{$\begin{array}{l}\text { First Lean } \\
\text { tissue mass }\end{array}$} & \multirow[t]{2}{*}{0.37} & First Age & 0.37 & \multirow{2}{*}{$\begin{array}{l}\text { First Lean } \\
\text { tissue mass }\end{array}$} & \multirow[t]{3}{*}{0.44} \\
\hline$R^{2}$ from first & $\begin{array}{r}\text { Second Age } \\
22\end{array}$ & 0.54 & $\begin{array}{r}\text { Second ALES } \\
29\end{array}$ & 0.57 & & & $\begin{array}{r}\text { Second ALES } \\
14\end{array}$ & 0.51 & & \\
\hline $\begin{array}{l}\text { Cumulative total explained } R^{2} \text { with } \\
\text { first and second predictors (\%) }\end{array}$ & 29 & & 33 & & 14 & & 26 & & 18 & \\
\hline
\end{tabular}

ALES, absolute leg extension strength.

${ }^{a}$ The entry order of predictor variables into the MLRA model is as indicated by "first" and "second." The beta coefficients, level of significance ( $P$ values), and cumulative explained variance $\left(R^{2}\right)$ for included variables are presented for each BMD site.

BMD in triathletes compared with the weight supported athlete groups, the swimmers and the cyclists. The significantly higher adjusted leg BMD and trend toward higher FN BMD in triathletes supports our first hypothesis of intermediate level regional BMD, associated with the additional weight bearing (running) loading in this sport. Failure to detect additional regional BMD differences between the triathletes and the other sport groups may be explained by the allotment of training time to weight supported and weight bearing activities in triathlon. Triathletes devoted over 50\% (8 h) of their training time to swimming and only about $25 \%(4 \mathrm{~h})$ of this time to running.

As in previous studies (e.g., Fehling et al. (11)), our results suggest that swimming provides little if any general (TB) or regional (arm, leg) osteogenic stimulus, at least when assessed by BMD. Thus, it could be argued that the weightlessness of swimming might attenuate the otherwise positive benefits of the diverse and unusual strain patterns involved in triathlon. However, it also appears that this potential attenuation may be offset in the legs for triathletes, and BMD augmented by the superimposition (in addition to the $12 \mathrm{~h}$ of swimming and cycling training) of as few as $4 \mathrm{~h}$ of running per week, commencing shortly after menarche. Whether this trend toward augmented leg BMD in triathletes is attributable to the inclusion of this additional element of weight bearing loading, to increased cumulative training volume, or to selection bias cannot be determined with this study design. Nevertheless, including an element of weight bearing activity postmenarche appears to be associated with nonsignificantly augmented regional (leg) BMD in triathletes compared with swimmers and cyclists.

Previous research (15) has failed to demonstrate a positive association between cycling and BMD. Our study reports BMD in elite adolescent female cyclists, and in contrast to previous studies, there was a trend (nonsignificant) for higher unadjusted BMD at the FN, LS, and TB for the cyclists compared with controls and swimmers. These differences were less evident, however, after adjustment for YPT, YSM, and LTM, with differences persisting only in comparison with swimmers. The trend toward higher unadjusted BMD for cyclists in our study, especially compared with controls, appears to conflict with previous findings (15). Discrepancies between studies may be explained by differences in age, training volume, and the possible deleterious effects of performance-enhancing drugs on BMD, especially in older professional athletes. Nevertheless, the lack of significant differences in BMD between cyclists and controls suggests that cycling does not provide a strong osteogenic stimulus for bone mineral accrual in adolescent females.

There is substantial variation in commencement ages for participation in various competitive youth sport programs, and this was evident in the present study. It was not possible, therefore, to match all groups for sport specific training history, and to control, a priori, for this potential confounding factor in our study design. Elite competition and specialized training in cycling and triathlon usually do not occur until after puberty, whereas swimmers and runners tend to specialize at an earlier age. In this study, runners began training earlier (premenarcheally and around $12.6 \mathrm{y}$ of age on average) and had specialized in their sport for a greater number of years than triathletes and cyclists. Increased cumulative sport specific weight bearing exposure, therefore, is a possible but improbable explanation for the higher BMD in runners, since the differences persisted even after statistical adjustment for the influences of YPT, YSM, and LTM. The earlier starting age of training for runners is another possible explanation for these differences. Although there is good evidence to suggest a greater influence of exercise on skeletal adaptations in immature compared with mature animals (16), there is no conclusive evidence of a more robust skeletal response to "identical" exercise programs within a particular stage of development during childhood. Furthermore, starting age of training and years of sport specific training history would have had little influence on the comparisons between swimmers and runners, as these groups differed the least for these variables. If anything, their slightly earlier starting age of training and longer sport specific training history would have conferred a BMD advantage to swimmers, and this clearly was not the case in the present study.

Given its cross-sectional design, we cannot discount selection bias as an alternative explanation for the observed differences in BMD among groups in our study. Several recent studies $(1,7,24)$ have proposed that similarities in BMD of the head, a relatively unloaded skeletal region during most sport activities, argue against selection bias in cross-sectional comparative studies of this nature. Skull BMD was similar across all groups in the present study, suggesting that observed differences in regional BMD 
among athletes were associated with sport specific loading patterns rather than selection bias. Additionally, dietary calcium intakes (assessed by 3-d food records) were similar among groups, so it is unlikely that differences in calcium status influenced our BMD comparisons.

Although BMD adaptations to mechanical loading are generally anatomically site-specific (4), they are also dependent on the achievement of a minimum effective strain to achieve a modeling response (12). Anatomically, swimming predominantly loads the arms and legs, but in this study, swimmers had nonsignificantly different arm BMD and the lowest leg BMD of all groups. This may be explained by the fact that the muscle tensile forces in swimming are not large enough to elicit positive regional skeletal adaptations on their own, or that they were insufficient to counter the influence of the nonweight bearing nature of this activity. The trend toward lower BMD of swimmers across all sites suggests that swimming, even if begun before puberty and done for an extended period, appears to be the least osteogenic (at least in terms of BMD) of the four sports examined in this study.

Since the athletes in this study were all involved in endurance events, the magnitude of the muscle contraction forces and the resultant site-specific bone strains during training and competition would be expected to be submaximal (13) and similar across sport disciplines. This was confirmed by the similarity in absolute and normalized strength measures among sport groups in the present study. The trend toward higher strength levels in athletes compared with controls probably reflects increased neuromuscular conditioning associated with the increased physical activity of training. The runners were the strongest of all groups in this study, and the only group that was significantly stronger than controls. Endurance running typically involves highrepetition, low-force muscle contractions (13), and these would not be expected to elicit significant strength gains. Runners in our study, however, were middle-distance specialists, and there may be a higher muscle force requirement for runners at these distances than at long-distance events.

The increased strength of the runners is not likely because of differences in supplemental strength training, as equal and small $(N=3-4)$ numbers of athletes from each sport reported partaking in this activity. Selection bias and inadequate statistical power because of relatively small samples and high variability in strength measures may also partially explain the pattern of observed strength differences in this study. These strength results suggest that the differences in total body and regional BMD among groups in the present study are more likely attributable to the differential sport specific weight bearing and nonweight bearing nature of the loading patterns and differences in ground reaction forces than to differences in bone strain levels associated with muscle contraction forces acting on bone.

Significant relationships between strength and BMD have been reported previously for young adult females (e.g., Madsen et al. (21) and Snow-Harter et al. (29)). To our knowledge, however, there are limited $(1,25)$ reports describing these relationships in adolescent females. Our results support previous findings of significant relationships between absolute leg ex- tension strength and BMD, and in part confirm our third hypothesis. Correlations were, however, only of low to moderate strength and weaker for normalized compared with absolute strength. These findings are consistent with those of Madsen et al. (21) for young adult females, and suggest that positive associations between absolute strength and BMD in simple bivariate relationships are most likely attributable to covariation with some unaccounted factor such as increased LTM that acts independently of the influence of normalized or approximated muscle-specific tension. Increased LTM might influence bone strain level and BMD, independent of muscle strength, via the mechanism of increased ground reaction and axial compressive forces. This is a plausible explanation in our study, where there was little difference in normalized strength of the leg muscles across sport groups. Whether this relationship and the above explanation apply to other athlete groups with more variable normalized or muscle-specific tension characteristics remains to be determined.

Our results also suggest that leg extension strength is more strongly associated with BMD across sites than leg flexion strength. These findings contrast with previous reports for adolescent males (24) and females (1), and may reflect either a greater reliance on and recruitment of extensor muscles, or differential muscle-induced bone strains resulting from differences in muscle attachment sites and the biomechanical actions of the knee flexor and extensor muscle groups during the execution of the sports examined in the present study.

Absolute muscle strength accounted for small yet significant increments in the explained variance for LS and leg BMD in the MLRA, suggesting that strength per se may be an important independent predictor of BMD at these sites in adolescent females. Part of this increment, however, may also be attributable to the residual influence of LTM on both BMD and muscle strength, since LTM did not enter as an independent predictor in either of these regression models. The failure of normalized strength to enter as a significant independent predictor for any of the BMD variables in the regression analyses and the lack of differences in leg BMD among normalized strength quartile groups, however, suggest that muscle strength is a relatively unimportant determinant of the differences in total and regional BMD observed in the present study.

Collectively, these observations suggest that bone strains induced by the nature of the loading condition and the magnitude of sport specific ground reaction forces may play a more important role than muscle tensile forces themselves in influencing osteogenic differences among elite adolescent female athletes. This at first seems to conflict with conventional theory, which claims that muscle force is the predominant determinant of osteogenic adaptations to mechanical loading during exercise. Our findings do not preclude, however, a significant influence of muscle tensile forces on BMD during the initial years of sport specialization, in the "adaptive" phase of training. Once adapted, however, one would expect from both the biological perspective of the mechanostat theory (13) and a statistical perspective of reduced variability in muscle strength a relatively weak or attenuated association between strength and BMD among athletes with similar strength profiles. In the absence of differences, or further increases in normalized 
strength, following the initial adaptation to training, it appears that the nature of the sport specific training loads and the magnitude of their associated ground reaction forces may be more important determinants of differentiation in BMD among adolescent female athletes than muscle contraction forces themselves.

In conclusion, BMD was highest in the weight bearing sport of running, and these results were consistent across all measurement sites. Although not statistically significant, there was a trend toward higher regional BMD in triathletes compared with swimmers and cyclists, suggesting a positive osteogenic influence of regional weight bearing loading exercise. Absolute muscle strength, although significantly correlated with BMD at most measurement sites, was neither a strong predictor nor an effective discriminator of leg BMD among sport groups after

\section{REFERENCES}

1. Alfredson, H., G. Hedberg, E. Bergstrom, P. Nordstrom, and R. LoRENTZON. High thigh muscle strength but not bone mass in young horse-back riding females. Calcif. Tissue Int. 62:497-501, 1998.

2. Blimkie, C. J., S. Rice, C. E. Webber, J. Martin, D. Levy, and C. L. Gordon. Effects of resistance training on bone mineral content (BMC) and density in adolescent females. Can. J. Physiol. Pharmacol. 74:1025-1033, 1996.

3. Blimkie, C. J. R., P. Chilbeck, and K. S. Davison. Bone mineralisation patterns: reproductive, endocrine, calcium, and physical activity influences during the lifespan. In: Perspectives in Exercise Science and Sports Medicine, Vol. 9: Exercise and the Female-A Lifespan Approach, O. Bar-or, D. R. Lamb, and P. M. Clarkson (Eds.). Carmel, IN: Cooper Publishing Group, 1996, pp. 73-145.

4. Bourrin, S., S. Palle, R. Pupier, L. Vico, and C. Alexandre. Effect of physical training on bone adaptation in three zones of the rat tibia. J. Bone Miner. Res. 10:1745-1752, 1995.

5. Burr, D. B. Muscle strength, bone mass, and age-related bone loss. J. Bone Miner. Res. 12:1547-1551, 1997.

6. Corbin, C. B. The untracking of sedentary living: a call for action. Ped. Ex. Sci. 13:347-356, 2001.

7. Courteix, D., E. Lespessailles, P. Obert, and C. L. Benhamou. Skull bone mass deficit in prepubertal highly-trained gymnast girls. Int. J. Sports Med. 20:328-333, 1999.

8. Courteix, D., E. Lespessailles, S. L. Peres, P. Obert, P. Germain, and C. L. Benhamou. Effect of physical training on BMD in prepubertal girls: a comparative study between impact-loading and non-impact-loading sports. Osteoporos. Int. 8:152-158, 1998.

9. Day, S. J., and D. F. Graham. Sample size and power for comparing two or more treatment groups in clinical trials. BMJ 299: 663-665, 1989.

10. Dyson, K., C. J. Blimkie, K. S. Davison, C. E. Webber, and J. D. ADACHI. Gymnastic training and bone density in pre-adolescent females. Med. Sci. Sports Exerc. 29:443-450, 1997.

11. Fehling, P. C., L. Alekel, J. Clasey, A. Rector, and R. J. Stillman. A comparison of bone mineral densities among female athletes in impact loading and active loading sports. Bone 17:205210, 1995.

12. Frost, H. M. A determinant of bone architecture: the minimum effective strain. Clin. Orthop. 175:286-292, 1983.

13. Frost, H. M. Why do marathon runners have less bone than weight lifters? A vital-biomechanical view and explanation. Bone 20:183-189, 1997.

14. Heinonen, A., P. Oja, P. Kannus, et al. BMD in female athletes representing sports with different loading characteristics of the skeleton. Bone 17:197-203, 1995.

15. Heinonen, A., P. Oja, P. Kannus, H. Sievänen, A. Mänttäri, and I. VUORI. BMD of female athletes in different sports. Bone Miner. 23:1-14, 1993. normalization for leg LTM. These results suggest that the nature (weight bearing exercise with longitudinal compressive strain) of the loading pattern and the magnitude of associated sport specific ground reaction forces may be more important determinants of BMD than either the anatomical distribution of loads or muscle strength in the adolescent female athlete groups investigated in this study. Prospective controlled exercise trials, involving different types and combinations of loading conditions, with varying magnitudes of ground reaction and muscle contraction forces, will ultimately be required to confirm the observations in this cross-sectional study.

Address for correspondence: C. J. R. Blimkie, FACSM, Department of Kinesiology, McMaster University, Hamilton, Ontario, Canada, L8S 4K1; E-Mail: blimkie@mcmail.mcmaster.ca.

16. Järvinen, T. L., P. Kannus, H. Sievänen, P. Jolma, A. Heinonen, and M. JäRVINEN. Randomized controlled study of effects of sudden impact loading on rat femur. J. Bone Miner. Res. 13:14751482, 1998.

17. Judex, S., T. S. Gross, and R. F. Zernicke. Strain gradients correlate with sites of exercise-induced bone-forming surfaces in the adult skeleton. J. Bone Miner. Res. 12:1737-1745, 1997.

18. LANYON, L. E. Functional strain in bone tissue as an objective, and controlling stimulus for adaptive bone remodelling. J. Biomech. 20:1083-1093, 1987.

19. Louis, O., K. Demeirleir, W. Kalender, et al. Low vertebral bone density values in young adult non-elite female runners. Int. J. Sports Med. 2:214-217, 1991.

20. Lu, P. W., J. N. Briody, G. D. Ogle, et al. BMD of total body, spine, and femoral neck in children and young adults: a crosssectional and longitudinal study. J. Bone Miner. Res. 9:14511458, 1994.

21. Madsen, K. L., W. C. Adams, and M. D. Van Loan. Effects of physical activity, body weight and composition, and muscular strength on bone density in young women. Med. Sci. Sports Exerc. 30:114-120, 1998.

22. Moen, S. M., C. F. Sanborn, N. M. Dimarco, et al. Lumbar BMD in adolescent female runners. J. Sports Med. Phys. Fitness 38: 234-239, 1998.

23. Nordstrom, P., U. Pettersson, and R. Lorentzon. Type of physical activity, muscle strength, and pubertal stage as determinants of BMD and bone area in adolescent boys. J. Bone Miner. Res. 13:1141-1148, 1998.

24. Nordström, P., K. Thorsen, E. Bergström, and R. Lorentzon. High bone mass and altered relationships between bone mass, muscle strength, and body constitution in adolescent boys on a high level of physical activity. Bone 19:189-195, 1996.

25. Rice, S., C. J. Blimkie, C. E. Webber, et al. Correlates and determinants of BMC and density in healthy adolescent girls. Can. J. Physiol. Pharmacol. 71:923-930, 1993.

26. Rutherford, O. M. Spine and total body BMD in amenorrheic athletes. J. Appl. Physiol. 6:2904-2908, 1993.

27. Schiessl, H., H. M. Frost, and W. S. Jee. Estrogen and bonemuscle strength and mass relationships. Bone 22:1-6, 1998.

28. SсHÖNAu, E. The development of the skeletal system in children and the influence of muscular strength. Horm. Res. 49:27-31, 1998.

29. Snow-Harter, C., M. Bouxsein, B. Lewis, S. Charette, P. WeinSteIN, and R. MARCus. Muscle strength as a predictor of BMD in young women. J. Bone Miner. Res. 5:589-595, 1990.

30. Snow-Harter, C., M. L. Bouxsein, B. T. Lewis, D. R. Carter, and R. MARCus. Effects of resistance and endurance exercise on bone mineral status of young women: a randomized exercise intervention trial. J. Bone Miner. Res. 7:761-769, 1992. 